\title{
ESTIMATING DEGRADATION GROWTH RATE AND TIME OF COMPONENT REPLACEMENT FROM LIMITED INSPECTION DATA USING MIXED-EFFECTS MODELLING
}

\author{
Mikko Jyrkama ${ }^{1}$, Mahesh Pandey ${ }^{2}$, and Ming $\mathbf{L i}^{3}$ \\ ${ }^{1}$ Research Associate, University of Waterloo, Waterloo, ON, Canada (mikko.jyrkama@uwaterloo.ca) \\ ${ }^{2}$ Professor, University of Waterloo, Waterloo, ON, Canada \\ ${ }^{3}$ Senior Technical Expert, Ontario Power Generation, Pickering, ON, Canada
}

\begin{abstract}
This paper presents the assessment of flow accelerated corrosion (FAC) of piping in the primary heat transport system of a nuclear reactor. While the degradation is known to proceed linearly with time, the estimation of the corrosion wear (i.e., wall thinning) rate is impacted by uncertainties in the inspection data, limited number of repeat observations, and the uncertain initial thickness of the piping components. The degradation model formulated in this study is based on the linear mixed-effects regression (LMER) approach, that considers both the population level (i.e., fixed) effects, as well as the individual pipe (i.e., random) components of thinning rate and initial wall thickness. The results show how the mixed-effects approach is ideally suited for pooling the relatively few data from individual components across the much larger pipe population (and sub-populations), to obtain more robust and comprehensive estimates of degradation growth rates and times of component replacement. The methodology not only reduces the uncertainty in prediction for individual pipes with repeat inspection data, but also allows credible predictions to be made for components with very limited information (e.g., one or two data points), including the uninspected population.
\end{abstract}

\section{INTRODUCTION}

The structural integrity and fitness for service assessment of engineering structures and components is challenged by various degradation mechanisms, such as corrosion, fatigue and creep. As a result, periodic inspections are carried out to detect the onset and occurrence of degradation and monitor its growth over time. However, because of the considerable variability associated with the degradation processes and the influence of other factors, such as material properties, operational stresses, environmental conditions, and errors in the inspection data, the analysis of system and component reliability over time is highly uncertain.

This paper presents the assessment of flow accelerated corrosion (FAC) of piping in the primary heat transport system of a nuclear reactor. Previous studies have shown the FAC wall thickness loss to proceed linearly with time, allowing simple linear regression to be applied to pipes with repeat data. However, many of the piping components have only been inspected once or twice, and even for pipes with several inspections, it is not possible to obtain a robust estimate for the time of replacement (and particularly the lower and upper uncertainty bounds) using the standard regression approach.

An additional and significant complicating factor in the assessment, particularly for pipes with limited number of inspections, is the uncertain and unknown initial wall thickness. No inaugural or initial measurements are available for the various pipe geometries with different wall thickness profiles. 
Rather than analyzing the degradation rate and time of replacement of each individual pipe in isolation, the methodology developed in this study considers all of the inspection data from the entire pipe population (or sub-populations) in a combined approach. The degradation model used in this study is based on the linear mixed-effects regression (LMER) approach. The "mixed-effects" refers to the fixed and random effects, where the fixed effects represent the population level regression slope (i.e., thinning rate) and intercept (i.e., initial wall thickness), while the random effects capture the degree of variation and correlation of each individual pipe from the population level estimates. The main advantage of the mixed-effects approach is the ability to reduce uncertainty in the predictions by pooling information from individual components across the larger population (and groups of sub-populations).

The next section provides the background information about the piping system considered in this study, while the model methodology is presented in the following section. The subsequent study results clearly demonstrate the main benefits of the methodology, which are then summarized in the last section of the paper.

\section{SYSTEM DESCRIPTION}

\section{Feeder Piping System}

As shown in Figure 1, feeder piping circulates the primary pressurized heavy water coolant between the fuel channels in a CANDU reactor core and the steam generators. Each fuel channel is connected with an inlet and an outlet feeder pipe through a Grayloc coupling, resulting in a total of 380 to 480 inlet and outlet feeders depending on the reactor design. The feeder pipes are made of SA106 Grade B carbon steel, with the outlet feeders consisting of 2 inch $(51 \mathrm{~mm})$ and 2.5 inch $(64 \mathrm{~mm})$ diameter pipes with 5.5 $\mathrm{mm}$ and $7.0 \mathrm{~mm}$ nominal wall thicknesses, respectively. The feeder piping was formed by bending the individual pipes into various geometries, resulting in variable wall thickness profiles at the bends. No inaugural (i.e., time of installation or initial) wall thickness measurements are available for the pipes.

This study considers the wall thickness data from a multi-unit nuclear station, consisting of four (nominally) identical reactors each with a total of 480 inlet and outlet feeder pipes.

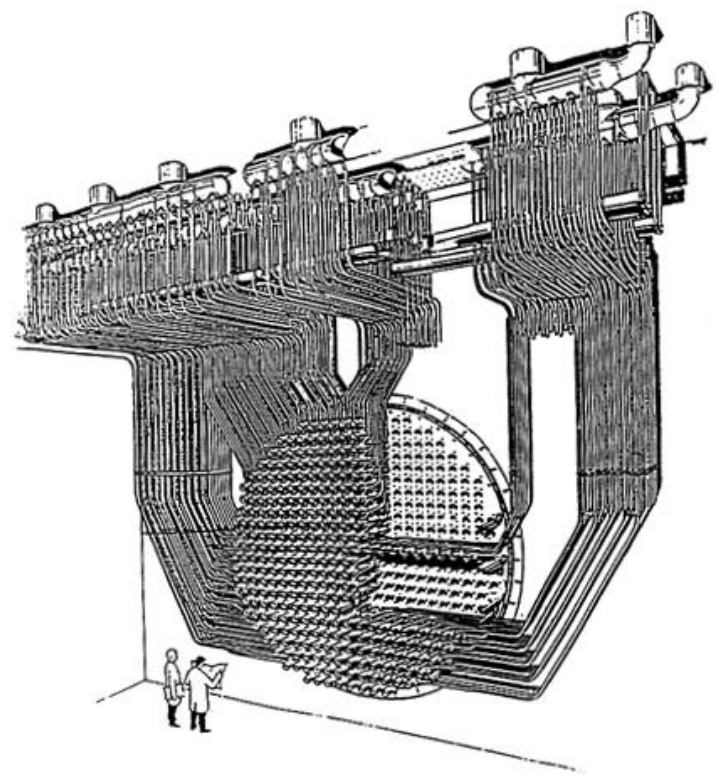

Figure 1. Schematic of CANDU feeder piping system layout. Some feeder pipes have been removed for clarity (image courtesy of CANTEACH - http://canteach.candu.org). 


\section{Degradation Mechanism}

Flow accelerated corrosion (FAC) is the major form of degradation impacting the operating life of feeder piping in CANDU reactors (Slade and Gendron, 2005; Jin et al., 2008; Chung et al., 2010). The rate of wall loss is highest in the tight-radius bends near the outlet feeder Grayloc weld. The FAC process is affected by many factors, including water chemistry (primarily $\mathrm{pH}$ ), fluid velocity and flow rate (i.e., pipe geometry), material composition (chromium content) and operating temperature (Burrill and Cheluget, 1999). The average operating temperature at the outlet feeders is around $310^{\circ} \mathrm{C}$, while the heavy water coolant $\mathrm{pH}$ is generally maintained between 10.0 and 10.3 .

The extent of FAC induced wall thickness loss is measured using bracelet type ultrasonic (UT) tools that are mounted on the outside surface of a feeder pipe and then rotated circumferentially or pulled/pushed axially along the length of the pipe. As a result, the measurement error or uncertainty associated with the inspection data is due primarily to the manual operation of the tools (i.e., spatially unreferenced grids) and probe signal loss and coverage issues (Jyrkama and Pandey, 2012; 2017).

\section{Degradation Model}

Previous studies have shown that the FAC induced wall thickness loss exhibits a strong linear correlation with time (Jin et al., 2008; Chung et al., 2010; Jyrkama and Pandey, 2012). Therefore, it is reasonable to assume that the wall thickness loss can be described by the linear random rate model of degradation, implying that each feeder pipe has a different, but constant (and unknown) thinning rate over time.

Figure 2 shows the minimum wall thickness data over time for the first bend of an example 2 inch diameter feeder pipe from one of the four reactors at the multi-unit station. The solid orange line represents the mean prediction from simple linear regression of the repeat data, while the shaded area corresponds to the $95 \%$ prediction interval.

As shown by Figure 2, the regression fit is quite good, with an $\mathrm{R}^{2}$ of 0.986 , and an estimated thinning rate (i.e., slope of the regression line) of $0.070 \mathrm{~mm} /$ year. However, because of the small sample size (i.e., only three inspections), the prediction interval is extremely wide for this pipe, making the estimation of the time of replacement highly uncertain.

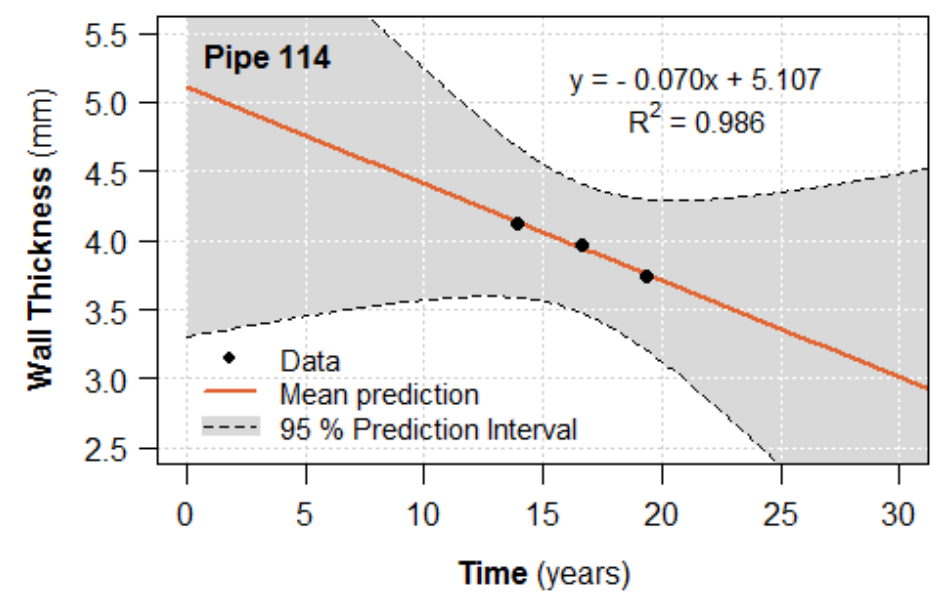

Figure 2. Minimum wall thickness over time at the first bend of a 2 inch diameter type $M$ feeder pipe 114, including the mean linear regression prediction and the $95 \%$ prediction interval. 
Comparison of numerous wall thickness scans from the different reactors has shown that the pattern of wall thickness loss, in terms of the general shape and location, is directly related to the underlying pipe geometry. This means that feeder pipes with the same geometry will generally experience FAC related wall loss at the same locations (due to similar flow conditions), regardless of the reactor unit. The actual depth, however, is variable as a result of specific local and global conditions.

Figure 3 shows the fitted regression lines of all type $M$ feeder pipes with first bend repeat data (i.e., $\geq 3$ inspections) at the multi-unit station. The first bend geometry of the type $\mathrm{M}$ feeders is also included in Figure 3. Each unit has a total of 16 type $M$ pipes, for a total of 64 pipes at the station. Figure 3 also includes the linear fit (highlighted as a solid black line) of pipe 114 shown in Figure 2. The regression $\mathrm{R}^{2}$ of all the fits in Figure 3 is $\geq 0.98$. The highest number of repeat observations is five.
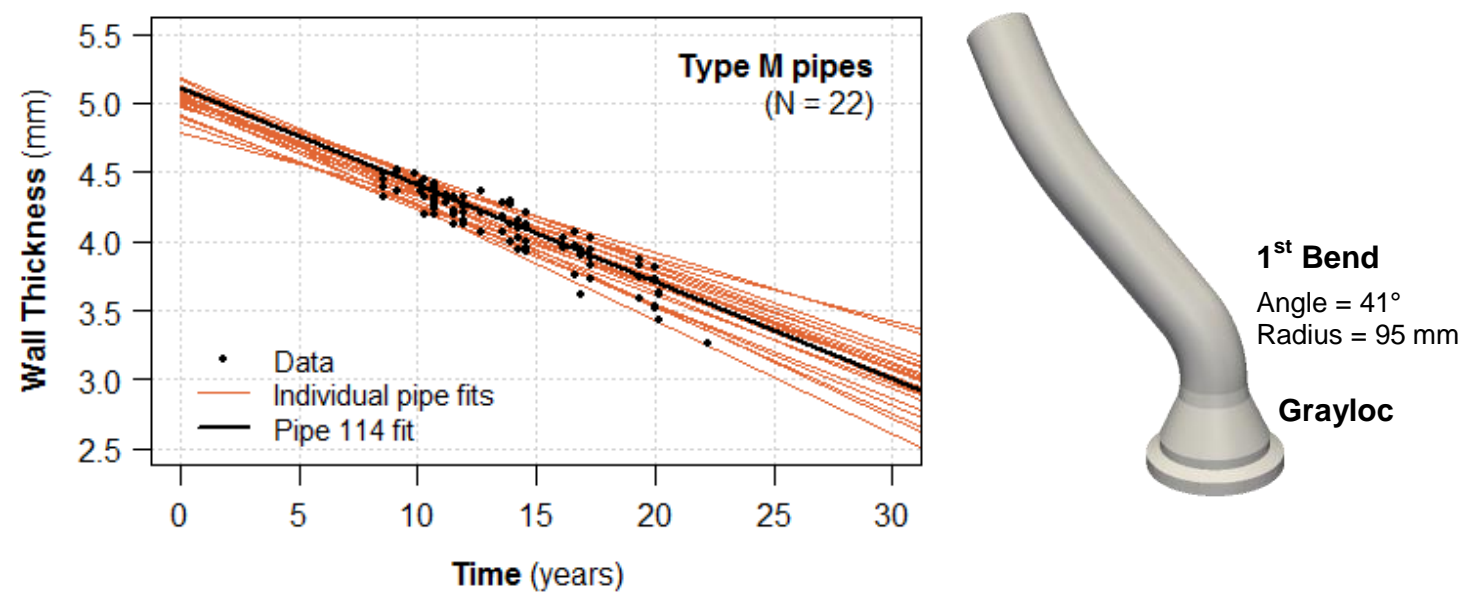

Figure 3. Fitted linear regression lines of all type $M$ feeder pipes with repeat data ( $\geq 3$ inspections). The linear fit of pipe 114 from Figure 2 is highlighted by the solid black line.

As shown by Figure 3, the overall trends are quite similar as expected, with small deviations in slopes (i.e., thinning rates) due to specific local effects (i.e., chemistry, temperature, flow conditions, etc.). Similar results are found for other pipe geometry groups at the station. The initial thickness at the first bend (i.e., intercept at time zero) is around $5.0 \mathrm{~mm}$, which is $0.5 \mathrm{~mm}$ less than the nominal initial thickness of $5.5 \mathrm{~mm}$. This is due to the bend extrados being thinner as a result of the bending.

As shown earlier in Figure 2, applying simple linear regression to the results from a single pipe is subject to significant uncertainty due to the small sample size. Moreover, most of the type M pipes (i.e., total of 42 pipes that were not included in Figure 3) have data from only one or two inspections that cannot be modelled using the simple regression approach. Therefore, the objective of this study is to use the data from all the pipes in a combined mixed-effects approach for estimating the degradation growth rates and times of replacement of all the pipes in the reactor.

\section{METHODOLOGY}

The main objective of mixed-effects (also referred to as multilevel, or hierarchical) modelling is to combine information from individual components (within a particular sub-group or level) to estimate the individual and population (and sub-group) level results. These types of models are often applied to ecological and biological data that can have many grouping factors, such as populations, species, treatments, sites, etc. (Gelman and Hill, 2007). Example applications to the degradation analysis of engineering components are provided in e.g., Yuan and Pandey (2009) and Shahraki et al. (2017). 


\section{Linear Mixed-Effects Regression (LMER)}

Linear mixed-effects regression (LMER) is the extension of simple linear regression to include the slope and/or intercept for the individual components (i.e., random effects) and the population (i.e., fixed effects). Assuming the wall thickness loss in the piping components is linear in time we can write

$$
y_{i j}=\beta_{0}+\beta_{1} t_{i j}+b_{0 i}+b_{1 i} t_{i j}+e_{i j}
$$

where $y_{i j}$ and $t_{i j}$ are the wall thickness and inspection time of the $i^{\text {th }}$ component at outage $j$, respectively, $\beta_{0}$ and $\beta_{1}$ denote the initial thickness and the wall thinning rate of the pipe population (or group), respectively, $b_{0 i}$ and $b_{1 i}$ represent the (random) initial thickness and wall thinning rate of the $i^{\text {th }}$ component, respectively, and $e_{i j}$ is the error term associated with the regression.

As shown in Equation 1, the wall thickness of a particular piping component is dependent not only on the component specific initial thickness and thinning rate, but also on the overall population (or group) level results. Conceptually, the so called "random effects" for a particular component represent the deviations in intercept and slope of that component's time trend from the population values. It is evident that the resultant estimates will be influenced by the sample size for each component (i.e., the number of repeat inspections over time). The results will also depend on the degree of linearity (i.e., quality of fit) of the repeat observations. For example, few repeat data with significant scatter (i.e., departure from linearity) will only have a limited impact, leaving the pipe specific predictions closer to the population values, whereas for a pipe with multiple measurements along a linear path, the predictions will be much closer to the pipe specific values.

In this study, the two-level (or two-stage) mixed-effects model in Equation 1 is applied at the pipe geometry group level, corresponding to the data for the type $\mathrm{M}$ geometry group shown in Figure 3. As shown in Figure 3, assuming both a random (i.e., different, but constant) slope and intercept for each pipe within the group appears reasonable, and is consistent with the general observations of the FAC process. The model could also easily be extended to the reactor level (across all the different pipe geometry groups) by adding additional higher level terms to Equation 1.

\section{Model Fitting}

The mixed-effects approach is ideally suited for modelling the degradation data in the piping system containing sub-groups of pipes with identical geometries. For illustration, the LMER model in Equation 1 was fitted only to the data for the type $M$ feeder piping shown in Figure 3. In addition to the 22 pipes with repeat data ( $\geq 3$ inspections), the remaining 42 pipes with only a single or two inspections were also included in the model fit.

The so-called unbalanced longitudinal data (i.e., repeat inspection of individual components at different times) was fitted using the lme 4 package in the software R (Bates, 2010). The results were obtained using the restricted maximum likelihood estimation (REML) approach, which provides consistent estimates of the model parameters. The prediction intervals are not possible to compute analytically, therefore, they were obtained using a simulation approach available in the merTools package in R. Additional information regarding mixed models and their fitting is found in e.g., Gelman and Hill (2007), Galecki and Burzykowski (2013), and West et al. (2015).

The key assumptions in the model are that the random effects and the regression errors are independent and follow Normal distributions, while the measurements within each individual piping component are correlated. 


\section{RESULTS AND DISCUSSION}

Figure 4 shows the fitted mean trends for each of the 22 type $M$ feeder pipes included in Figure 3, as well as two additional pipes, i.e., pipes 147 and 340, having one and two inspections, respectively, for comparison. Note that the results for pipe 114, shown earlier in Figure 2, are also included in Figure 4.

The orange lines in Figure 4 indicate the simple linear regression fits based purely on the data available for each individual feeder pipe, equivalent to Figure 3. Note that the individual fits are often not clearly visible, as they are very close to the mixed-effects results (blue lines). The green lines in Figure 4 represent the simple linear regression of all of the inspection data from all the type $\mathrm{M}$ pipes together as a single combined group, resulting in an intercept of $5.14 \mathrm{~mm}$ and a slope of $-0.074 \mathrm{~mm} / \mathrm{year}$. These lines are identical in each panel, allowing a direct comparison with the population level results. The blue lines in Figure 4 represent the mean prediction from the mixed-effects LMER model for each pipe.

Note that there is no individual fit (orange line) for pipe 147, since it only has a single observation of wall thickness. For pipes with repeat data, the deviation from linearity is assumed to be attributed to the probe measurement errors.

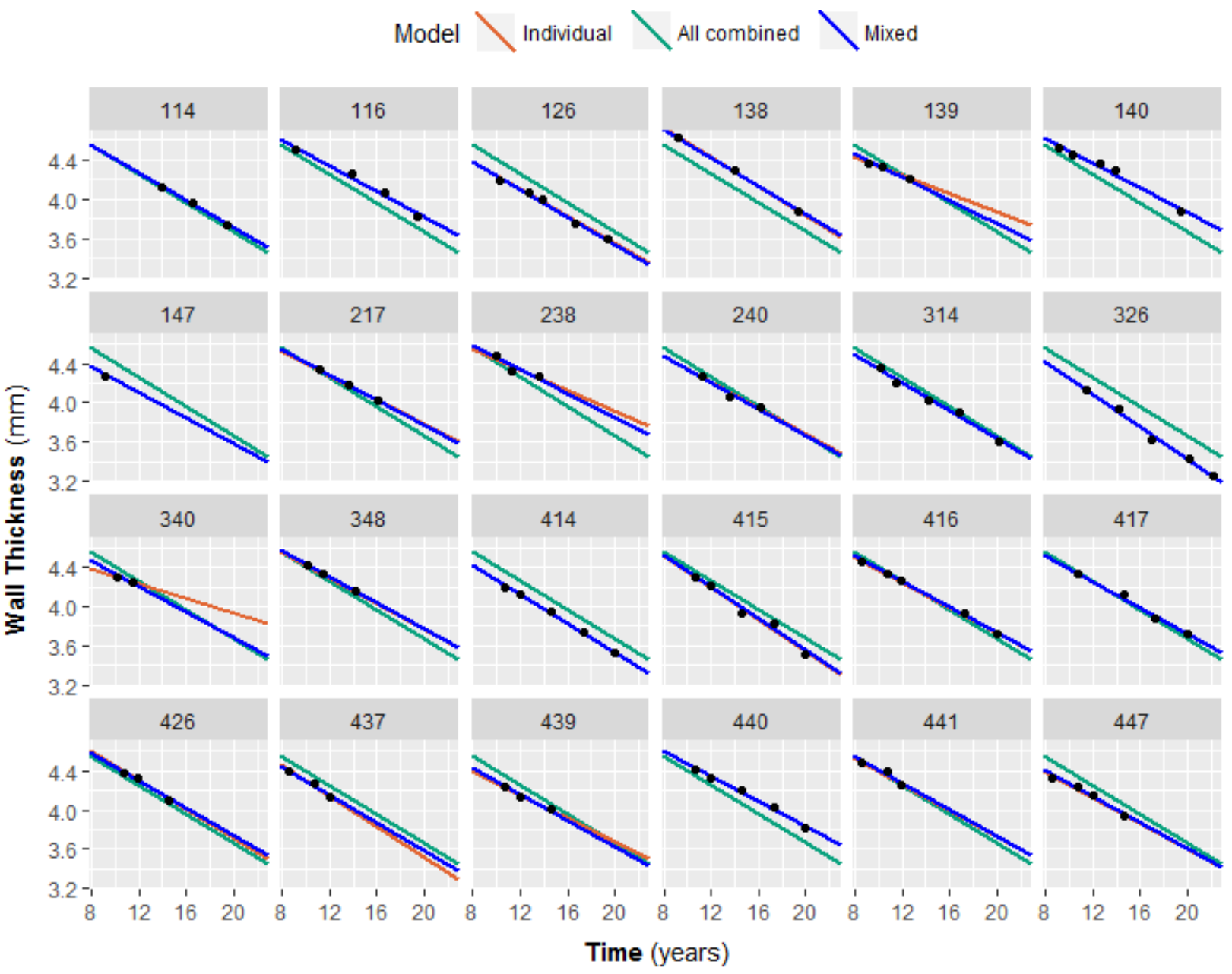

Figure 4. Mean trends for selected type $\mathrm{M}$ feeder pipes from individual regression, combined population (or group) level regression, and mixed-effects regression. The repeat inspection data for each pipe is indicated by the black dots. 
As shown in Figure 4, the slopes (i.e., thinning rates) of individual feeder pipes deviate from the group mean (green line) as a result of specific local conditions (e.g., chemistry, temperature, flow rate, etc.). Some pipes with fewer observations (e.g., 3 or less) have individual trends (orange lines) that deviate further from the group mean (likely due to measurement errors), however, the mixed-effects model results tend to be closer to the group average. This fundamental impact of the mixed-effects approach is further illustrated by the detailed prediction plots, shown in Figure 5.

Figure 5 shows the detailed LMER model predictions for four selected type $\mathrm{M}$ feeder pipes (included in Figure 4), including the $95 \%$ prediction intervals. The individual and combined group level mean trends are also included for comparison. Figure 5 illustrates how the mixed-effects model pulls more extreme estimates towards the overall average; the fewer and less linear the observations for a particular component, the greater the pull towards the average group estimate.

As shown in Figure 5, pipe 440 has a total of five repeat observations of wall thickness over time, with a slightly lower individual thinning rate $(0.063 \mathrm{~mm} / \mathrm{year})$ than the group average $(0.074 \mathrm{~mm} / \mathrm{year})$. Because of the larger number of data points and the very good individual fit $\left(\mathrm{R}^{2}=0.99\right)$, the mixed-effects model results are nearly identical to the individual model fit. The $95 \%$ prediction interval for the LMER model is also quite narrow. In contrast, pipe 139 and pipe 340 have much fewer data points ( 3 and 2, respectively), hence the LMER model fit is much closer to the overall group average (green line). The prediction intervals for the LMER model are also wider, and increase with distance away from the data.
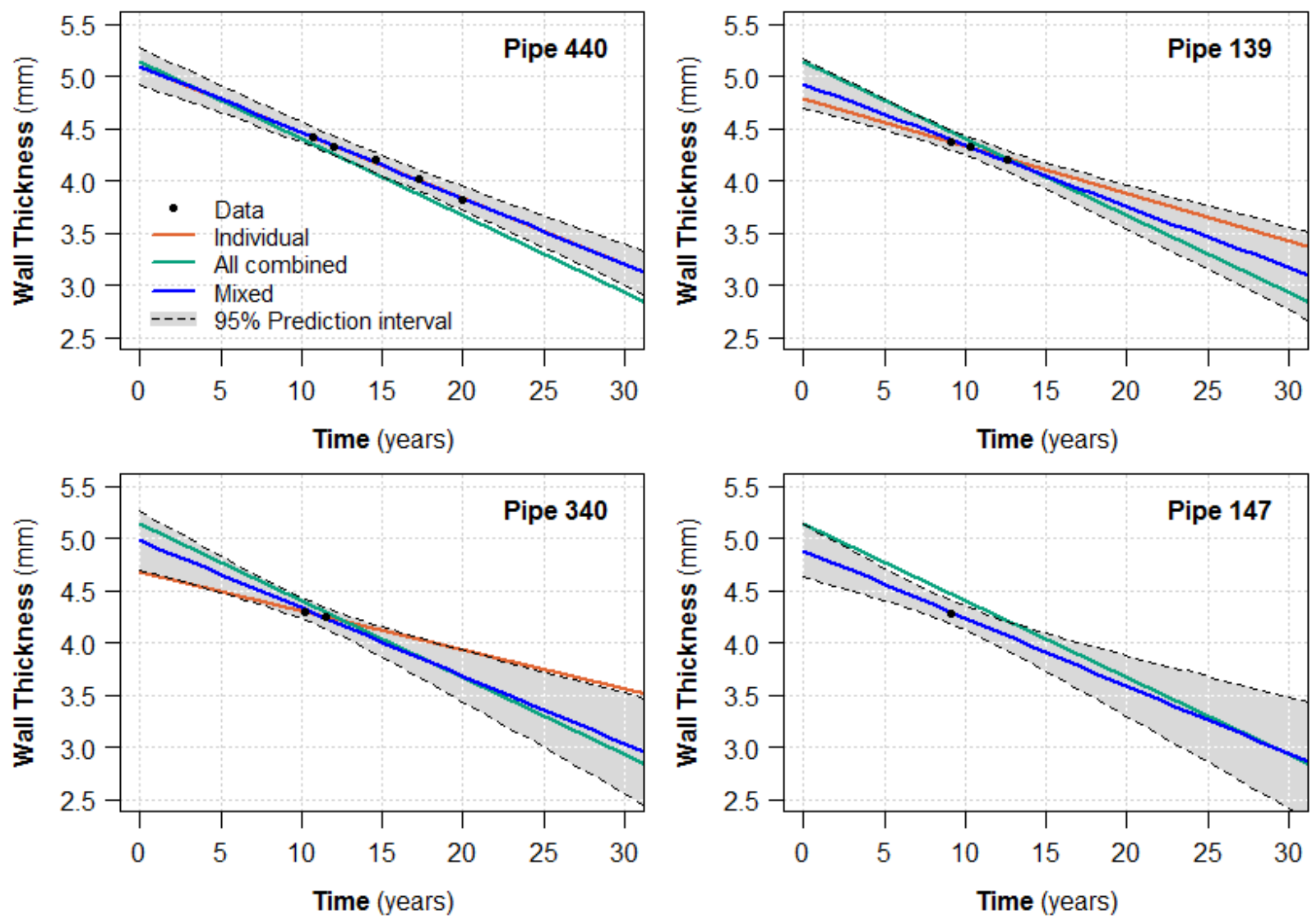

Figure 5. Mixed-effects regression model predictions for selected type $\mathrm{M}$ feeder pipes, including the $95 \%$ prediction intervals. The individual (orange lines) and combined group level means (green lines) are also included in the plots for comparison. 
The results for pipe 147 in Figure 5 also demonstrates how the mixed-effects model can be used effectively for estimating the thinning rate and component replacement time for pipes with only a single measurement. As expected, the width of the prediction interval increases away from the data point, and reflects the overall variation present among the type $M$ feeder pipes (shown in Figure 3).

The mixed-effects model can also be used to make predictions for any uninspected piping within the same group. In this case, the prediction for the uninspected components would simply correspond to the fixed effects part of the LMER model.

As a final comparison, Figure 6 shows the mixed-effects model prediction for pipe 114 that was discussed earlier in Figure 2. As shown in Figure 6, the mixed-effects model has a slightly lower estimated thinning rate $(0.073 \mathrm{~mm} /$ year $)$ than the group average. Most notably, however, the prediction interval for the LMER model is substantially narrower as compared to the simple regression of the individual data, shown earlier in Figure 2.

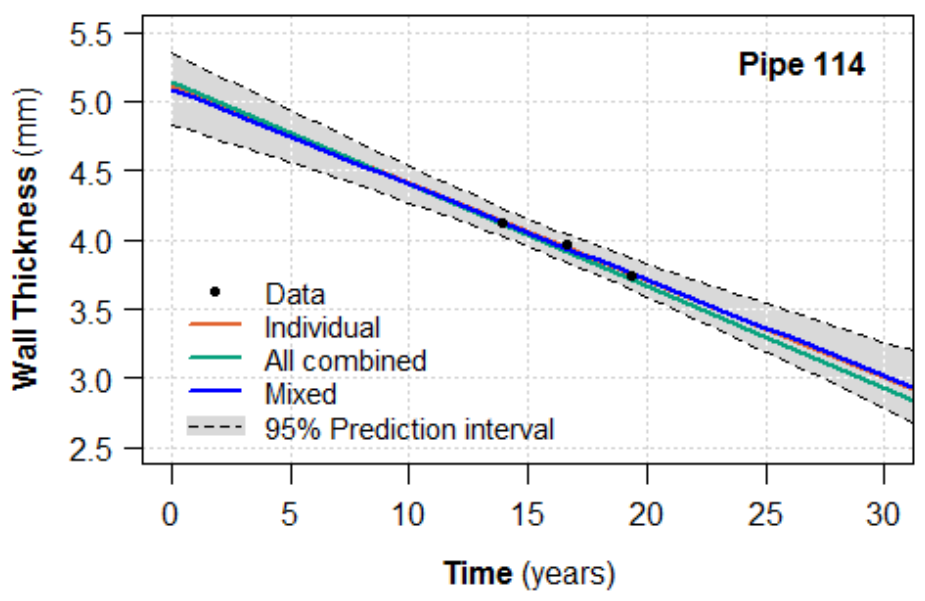

Figure 6. Mixed-effects regression model prediction for type $\mathrm{M}$ feeder pipe 114, including the $95 \%$ prediction interval. The individual (orange line) and combined group level mean (green line) are also included in the plot for comparison.

It is evident that the mixed-effects approach provides a highly effective method for estimating the degradation growth rate and time of replacement for groups of components with limited repeat inspection data. Pooling the data across individual components within the same group reduces the overall (i.e., sampling) uncertainty in the predictions, and allows effective predictions to be made for components with little or even no inspection data. Knowledge about the initial wall thickness is also not required. The impact of measurement errors, while minimized by the linear regression, is also included in the assessment. More extreme individuals/estimates (due to measurement errors) are pulled towards the overall group average, with the resulting shift depending on the number and linearity of the component observations.

\section{SUMMARY AND CONCLUSIONS}

This paper presented the assessment of flow accelerated corrosion (FAC) of piping in the primary heat transport system of a nuclear reactor, where that the estimation of the thinning rate and pipe replacement time was impacted not only by probe measurement errors and unknown initial pipe thicknesses, but also by the limited number of repeat observations. 
Because the FAC induced wall thickness loss was known to proceed linearly with time, the degradation model used in this study was based on the linear mixed-effects regression (LMER) approach. The mixed-effects approach provides a highly effective method for estimating the degradation growth rate and time of replacement for groups of components with limited repeat inspection data. The model considers both the population level (i.e., fixed) effects, as well as the individual pipe (i.e., random) components in the prediction. The benefits of the LMER method were illustrated by an example application to the degradation analysis of a group of feeder pipes at a multi-unit nuclear station. The model was fitted to the unbalanced longitudinal data using the software R.

The results of the study showed how pooling the data across individual piping components within the same group reduces the overall (i.e., sampling) uncertainty in the predictions, and allows effective predictions to be made for components with little or even no inspection data (i.e., uninspected components). Furthermore, reliable predictions can be made without knowledge of the initial wall thickness, and also include the impact of measurement errors. Components with more extreme observations (due to measurement errors) are pulled towards the overall group average, with the resulting shift depending on the number and linearity of the component observations; the fewer and less linear the observations for a particular component, the greater the pull towards the average group estimate.

In summary, the mixed-effects approach provides a highly effective and consistent method for combining all of the valuable (and often highly expensive) inspection data into a unified framework to obtain more robust and comprehensive estimates of degradation growth rates and times of replacement of all components in the pipe population.

\section{REFERENCES}

Bates, D.M. (2010). lme4: Mixed-effects modeling with R, Springer.

Burrill, K.A. and Cheluget, E.L. (1999). "Corrosion of CANDU Outlet Feeder Pipes", Report AECL11965, Atomic Energy of Canada Limited, Chalk River, Ontario, Canada.

Chung, H.-S. (2010). "A review of CANDU feeder wall thinning”, Nuclear Engineering and Technology, Vol. 42, No. 5, 568-575.

Galecki, A. and Burzykowski, T. (2013). Linear Mixed-effects Models Using R: A Step-by-step Approach, Springer.

Gelman, A. and Hill, J. (2007). Data Analysis Using Regression and Multilevel/Hierarchical Models, Cambridge University Press.

Jin, J.C., Carroll, B., and Awad, R. (2008). "FAC wall thinning of CANDU feeder piping", Proc. International Conference on Flow Accelerated Corrosion, Lyon, France.

Jyrkama, M.I. and Pandey, M.D. (2012). "Methodology for predicting flow-accelerated corrosion wear using unreferenced multiple inspection data", Nuclear Engineering and Design, Vol. 250, 317325.

Jyrkama, M.I. and Pandey, M.D. (2017). "Quantifying Probe Coverage Error in Feeder Thinning Assessment”, Proc. 37th Annual CNS Conference, June 4-7, 2017, Niagara Falls, ON, Canada.

Shahraki, A.F., Yadav, O. P. and Liao, H. (2017). "A Review on Degradation Modelling and Its Engineering Applications", International Journal of Performability Engineering, Vol. 13, No. 3, 299-314.

Slade, J.P. and Gendron, T.S. (2005). "Flow accelerated corrosion and cracking of carbon steel piping in primary water-operating experience at the Point Lepreau generating station", Proc. 12th International Conference on Environmental Degradation of Materials in Nuclear Power SystemsWater Reactors, The Minerals, Metals \& Materials Society.

West, B.T., Welch, K.B. and Galecki, A.T. (2015). Linear Mixed Models: A Practical Guide Using Statistical Software, $2^{\text {nd }}$ ed., CRC Press, Florida. 
Yuan, X.-X., Pandey, M.D. and Bickel, G.A. (2008). "A probabilistic model of wall thinning in CANDU feeders due to flow-accelerated corrosion", Nuclear Engineering and Design, Vol. 238, 16-24.

Yuan, X.-X. and Pandey, M.D. (2009). "A nonlinear mixed-effects model for degradation data obtained from in-service inspections", Reliability Engineering and System Safety, Vol. 94, 509-519. 